\title{
振荡压力烧结法制备高致密度细晶粒氧化锆陶瓷
}

\author{
李 双 ${ }^{1,2}$, 谢志鹏 ${ }^{1}$
}

(1. 清华大学 材料学院, 新型陶瓷与精细工艺国家重点实验室, 北京 $100084 ; 2$. 山东理工大学 资源与环境工程学 院, 淄博 255049)

摘 要: 针对重大工程和关键技术对高强韧性、高可靠性陶瓷部件的迫切需求, 本研究提出了适用于高性能陶瓷制 备的振荡压力烧结(Oscillatory Pressure Sintering, OPS)新方法, 并采用此种方法制备了高致密度、细晶粒、高强度 和高可靠性的氧化锆陶瓷。作为对比, 在相同的温度条件下分别采用无压烧结(Pressureless Sintering, PS)和热压烧 结(Hot Pressing, HP)制备了氧化锆陶瓷。SEM 研究表明振荡压力抑制了高温下氧化锆晶粒的快速生长, 统计表明 OPS 氧化锆的平均晶粒尺寸为 $251 \mathrm{~nm}$, 而 PS 氧化锆、 HP 氧化锆的平均晶粒尺寸分别为 $451 \mathrm{~nm}$ 和 $298 \mathrm{~nm}$ 。另外, 振荡压力引发的晶粒塑性形变和晶界滑移促进了晶界处闭气孔的排出, 使 OPS 氧化锆的体积密度提高到 $99.7 \%$ 。 氧化锆致密度的提高及晶粒细化使其断裂强度从 $1003 \mathrm{MPa}$ 提高到 $1572 \mathrm{MPa}$, Weibull 模数从 13 提高到 32。另外, 低 温老化实验表明 OPS 氧化锆的抗老化性能也得到显著提高。

关 键 词: 振荡压力; 晶粒细化; 强度韧性; 致密度

中图分类号: TB332 文献标识码: A

\section{Preparation of Zirconia Ceramics with High Density and Fine Grains by Oscillatory Pressure Sintering}

\author{
LI Shuang $^{1,2}$, XIE Zhi-Peng ${ }^{1}$
}

(1. State Key Laboratory of New Ceramics and Fine Processing, School of Materials Science and Engineering, Tsinghua University, Beijing 100084, China; 2. School of Resources and Environmental Engineering, Shandong University of Technology, Zibo 255049, China)

\begin{abstract}
To fulfill the demands of ceramic components with high strength, high toughness and high reliability in key projects, a novel oscillatory pressure sintering (OPS) method was proposed in this study. With this new method, zirconia ceramics with high relative density, fine grains, high fracture strength, and excellent reliability, were prepared. As a comparison, zirconia ceramics were sintered by pressure-less sintering (PS) method and hot pressing (HP) method at same temperature. SEM analysis shows that the oscillatory pressure inhibits the rapid grain growth of zirconia at high temperature. Statistic results indicate that the OPS zirconia presents fine grain size at $251 \mathrm{~nm}$, while the average sizes of PS and HP zirconia are 451 and 298 nm, respectively. In addition, the oscillatory pressure induces plastic deformation of grains and sliding of grain boundaries, facilitating elimination of closed pores at grain boundaries. As a result, the relative density of OPS zirconia reaches approximately $99.7 \%$. Due to the refinement of grains and the increase in relative density, the OPS zirconia presents an increase from $1003 \mathrm{MPa}$ to $1572 \mathrm{MPa}$ in flexural strength, and an increase from 13 to 32 in Weibull modulus. Furthermore, its resistance to low temperature degradation is also improved because of the
\end{abstract}


microstructure evolution.

Key words: oscillatory pressure; grain refinement; strength and toughness; relative density

高性能结构陶瓷具有高强度、高硬度、耐磨损、 耐腐蚀、耐高温和化学性质稳定等一系列优异性能, 在航空宇航、电子电器、机械制造、能源化工和生 命科学等领域受到的关注日益增强，应用领域也日 益广泛。然而, 多样化的应用环境也对陶瓷材料的 性能提出了更高的要求 ${ }^{[1-2]}$ 。例如, 航天火箭浴轮发 动机用的氮化硅陶瓷轴承 ${ }^{[3]}$ 、核聚变工程全超导托 卡马克装置中的氧化锆或氧化铝绝缘氦通道管 ${ }^{[4]}$ 、 半导体电子封装工业中用的寿命需要达到一百万次 的氧化锆陶瓷顶针、超高压活塞泵中的氮化硅陶瓷 柱塞等等, 都迫切需要有效控制材料的内部缺陷, 提高陶瓷材料的断裂强度及可靠性等指标。

陶瓷材料的强度和韧性等力学行为对组织结构 具有高度的敏感性, 尤其是气孔、微裂纹等各种微 观缺陷, 导致其实际力学性能远低于理论强度 ${ }^{[5-6]}$ 。 因此, 提高陶瓷材料的强度和韧性必须努力提高材 料的致密度使其达到或接近理论密度, 消除材料内 部的气孔、团聚体、微裂纹等微缺陷, 抑制晶粒生 长, 使晶粒尺寸和形状均匀化 ${ }^{[7-10]}$ 。在陶瓷材料制备 过程中, 烧结过程是控制材料微观性能的重要步骤, 该过程涉及温度、压力和气氛等因素及其调控, 因 此产生了常压烧结、真空烧结、压力烧结、气氛烧 结及各种各样的场辅助烧结技术 ${ }^{[11-15]}$ 。

通过施加压力提高陶瓷材料致密度的技术主要 有热压烧结(HP)、热等静压烧结(HIP)和放电等离子 烧结(SPS)三种。上述三种压力烧结方法都是在高温 烧结过程中对粉料施加外力, 通过这个外力赋予粉 料更高的烧结驱动力, 促进颗粒滑移、粘性流动等 机制, 从而制备具有较高力学性能和可靠性的陶瓷 构件。然而, 目前各种压力烧结技术所施加的轴向 压力皆为静态恒定压力。在烧结过程中, 静态压力 的局限性主要表现在: 烧结开始前, 恒定压力作用 下颗粒无法充满模具、颗粒无法滑移重排、颗粒团 聚体无法充分解聚, 使得素坏的堆积密度远小于其 理论堆积密度; 而在烧结后期, 静态压力下晶界处 残留闭气孔无法得到有效排除, 而闭气孔是制约结 构陶瓷力学性能提高的重要因素。因此, 采用静态 恒定压力烧结方法无法充分发挥压力对加速坏体致 密化、降低烧结温度、减少保温时间, 以及抑制晶 粒生长等的作用, 难以满足极端应用环境对陶瓷材 料的需求。

近几年来, 动态压力在粉体制备、材料加工和
部件考核中的应用日益广泛 ${ }^{[16-17]}$ 。Gourdin 等 ${ }^{[18]}$ 明 确提出动态压力在促进粉体致密化及提高材料强度 方面的作用显著。因此, 本研究将振荡压力应用于 氧化锆陶瓷的烧结, 研究了振荡压力对氧化锆陶瓷 微观结构与力学性能的影响; 通过对比无压烧结 (Pressureless Sintering, PS)氧化锆和热压烧结(Hot pressing, HP)氧化锆的结构与性能, 初步阐述了振 荡压力烧结(Oscillatory Pressure Sintering, OPS)技术 的特点。

\section{1 实验方法}

\section{1 实验原料}

实验所用的氧化锆粉体为商用氧化锆, 采用湿 化学法制备, 稳定剂选用氧化钎, 含量为 $3 \mathrm{~mol} \%$ 。 氧化锆粉体的形貌如图 1 所示, 平均粒径为 $80 \mathrm{~nm}$, 比表面积为 $10 \mathrm{~m}^{2} / \mathrm{g}$ 。

\section{2 制备方法}

采用自制振荡压力烧结设备制备振荡压力烧结 氧化锆，该设备可以对待烧粉体施加振幅与频率可 控的振荡压力, 从而提供较高的烧结驱动力, 促进 材料的致密化。振荡压力烧结设备的压力输出装置 如图 2(a)所示: 压力控制器 I 通过控制伺服阀 I 提供 压力 $P_{\mathrm{c}}, P_{\mathrm{c}}$ 为恒定压力, 数值较大; 压力控制器 II 通过控制伺服阀 II 提供压力 $P_{0}, P_{\mathrm{o}}$ 为振荡压力, 数 值较小; $P_{\mathrm{c}}$ 与 $P_{\mathrm{o}}$ 在压力点处有效耦合, 从而提供一 个振荡与频率可控的振荡压力。上述压力耦合方法 可以提供数值较大、振幅较小的振荡力, 避免单一

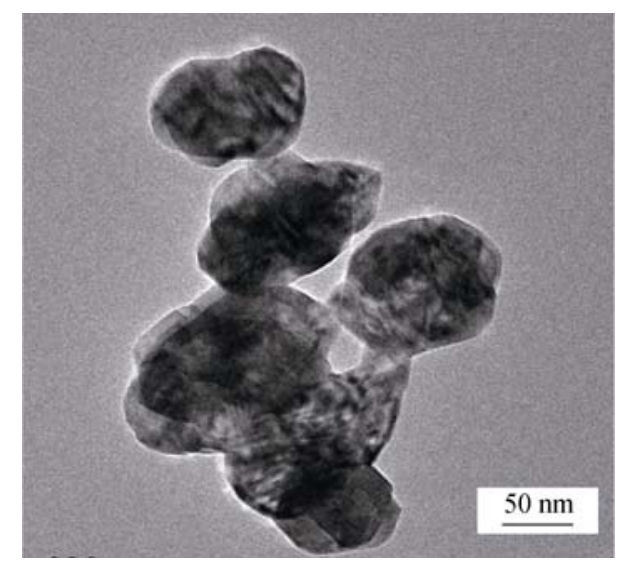

图 1 氧化锆粉体的 TEM 照片

Fig. 1 TEM images of zirconia particles 

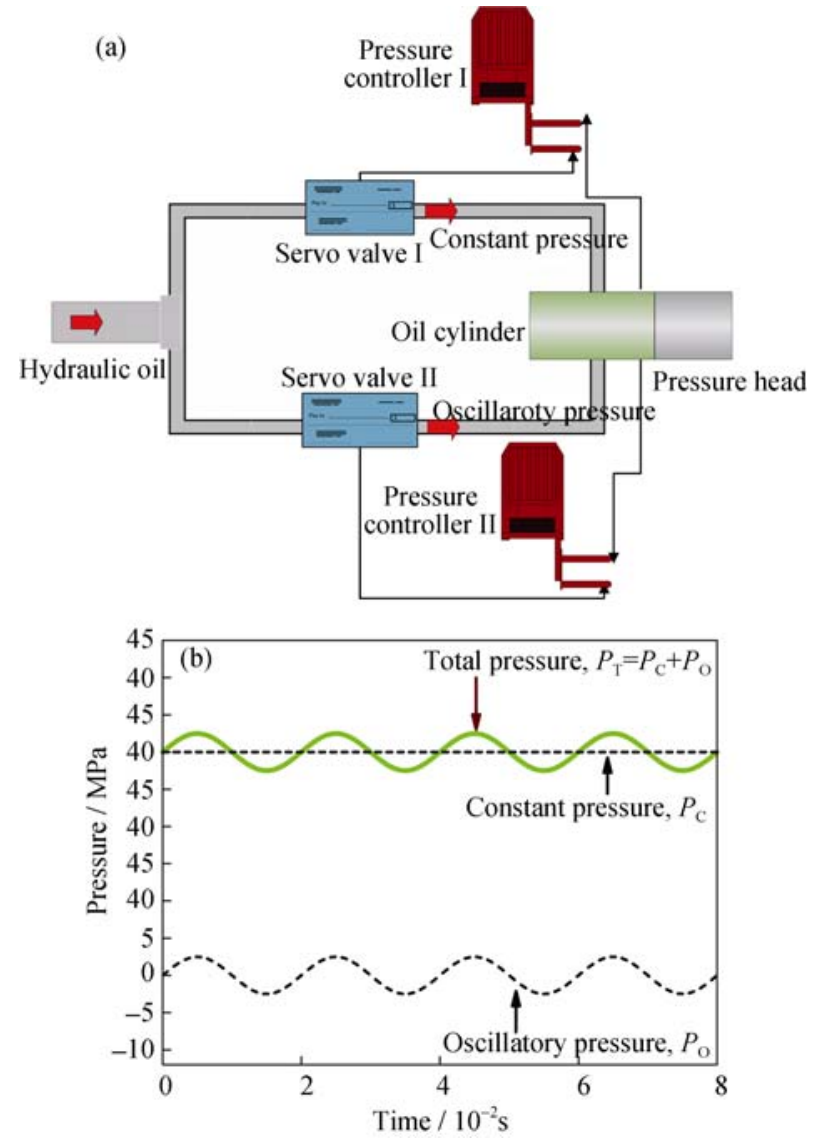

图 2 振荡压力耦合装置示意图(a)振荡压力耦合原理示意 图(b)

Fig. 2 Schematic of the coupling apparatus for oscillatory pressure (a) and the coupling mechanism for oscillatory pressure $\left(P_{\mathrm{T}}\right)$

振荡压力对待烧材料及模具造成的冲击损害。

采用下述工艺过程制备 OPS 氧化锆:

(1) 将氧化锆粉体装入石墨模具, 模具内径 $50 \mathrm{~mm}$;

(2) 向炉体通入氩气作为保护气体, 施加预压 力 $5 \mathrm{MPa}$ (恒定压力), 缓慢升温, 升温速率 $100^{\circ} \mathrm{C} / \mathrm{h}$;

(3) 当温度达到 $800^{\circ} \mathrm{C}$ 后保温 $30 \mathrm{~min}$, 排除粉体 中含有的结合剂等有机质;

(4) 将轴向压力(恒定压力)增加到 $15 \mathrm{MPa}$, 以 $100^{\circ} \mathrm{C} / \mathrm{h}$ 的升温速率继续升温到 $1000^{\circ} \mathrm{C}$, 保温 $30 \mathrm{~min}$;

(5) 将轴向压力(恒定压力)增加到 $30 \mathrm{MPa}$, 升 温到 $1450^{\circ} \mathrm{C}$, 升温速率 $50^{\circ} \mathrm{C} / \mathrm{min}$;

（6）温度达到 $1450^{\circ} \mathrm{C}$ 后施加振荡压力, 振荡压 力波形如图 2(b)所示, 压力变化范围为 27.5 32.5 MPa, 频率为 $50 \mathrm{~Hz}$ ，保温时间为 $45 \mathrm{~min}$;

(7) 保温完成后降温到 $900^{\circ} \mathrm{C}$, 降温速率 $100{ }^{\circ} \mathrm{C} / \mathrm{h}$, 并 缓慢卸压;

(8) 降温到 $900^{\circ} \mathrm{C}$ 后, 材料随炉自然冷却到室温。
作为对比, 采用相同的氧化锆原料进行无压烧 结和热压烧结。HP 氧化锆的制备方法与 OPS 氧化 锆相似, 只是在保温阶段一直施加 $30 \mathrm{MPa}$ 的恒定 压力。PS 氧化锆的制备步骤如下:

(1) 将氧化锆粉末压坏, 坏体尺寸 $\phi 50 \mathrm{~mm} \times$ $10 \mathrm{~mm}$, 压力 $30 \mathrm{MPa}$;

(2) 坏体干燥后移入马弗炉, 温度制度与 OPS 氧化锆相同。

\section{3 表征}

采用阿基米德法测量氧化锆的体积密度。采用 透射电子显微镜(JEOL-2100F; JEOL，Japan)观察氧 化锆粉体形貌及尺寸。采用扫描电子显微镜(LEO1503; LEO, Oberkochen, Germany)研究材料的微观 结构和晶粒尺寸，同时采用拦截法统计抛面处的晶 粒尺寸和晶粒分布。采用三点弯曲法测量材料的抗 弯强度, 试样尺寸为 $3 \mathrm{~mm} \times 4 \mathrm{~mm} \times 36 \mathrm{~mm}$, 跨距为 $30 \mathrm{~mm}$, 加载速率为 $0.5 \mathrm{~mm} / \mathrm{min}$ 。氧化锆的老化实 验执行 ISO13356-2008 标准 ${ }^{[19-20]}$ : 老化实验在不锈 钢高压反应釜中进行，将抛光后的试条置于反应釜 中, 加入蒸馏水，再将高压釜放入电热干燥箱中, 设定老化温度 $134^{\circ} \mathrm{C}$, 老化时间分别为 $6 、 12 、 18 \mathrm{~h}$ 。

\section{2 结果与讨论}

\section{1 微观结构}

实验制备的 PS、HP 和 OPS 三种氧化锆陶瓷的 断口形貌如图 3 所示。相同烧结温度下, 无压烧结 氧化锆已经具有较高的致密度, 但部分晶粒较为粗 大, 因此材料中粗颗粒与细颗粒共存, 断裂模式为 穿晶/沿晶混合型，如图 3(a)所示。在烧结过程中施 加 $30 \mathrm{MPa}$ 恒定压力后, 粉体的致密化速率明显加 大, 晶粒细化, 如图 3(b)所示。统计表明, HP 氧化锆 的平均晶粒尺寸为 $298 \mathrm{~nm}$, 而 PS 氧化锆的平均晶 粒尺寸为 $451 \mathrm{~nm}$ 。对待烧粉体施加 27.5 32.5 MPa 的振荡压力后, 氧化锆晶粒进一步细化, 统计表明 OPS 氧化锆平均晶粒尺寸为 $251 \mathrm{~nm}$, 此时断口处颗 粒表面比较光滑, 呈现为典型的沿晶断裂, 如图 3(c) 所示。同时, OPS 氧化锆晶粒尺寸的均匀性显著提 高, 如图 3(d)的抛光面所示。粒径统计分析表明, OPS 氧化锆晶粒分布在 200 350 nm 的较窄尺寸区 间内, 而 PS、HP 氧化锆中存在明显的晶粒粗化(部 分晶粒尺寸>500 nm)。

上述结果表明, 烧结过程中施加振荡压力可以 有效抑制氧化锆晶粒生长, 并且将晶粒尺寸控制在 较窄的尺寸区间内。根据 Coble 烧结理论, 烧结后 


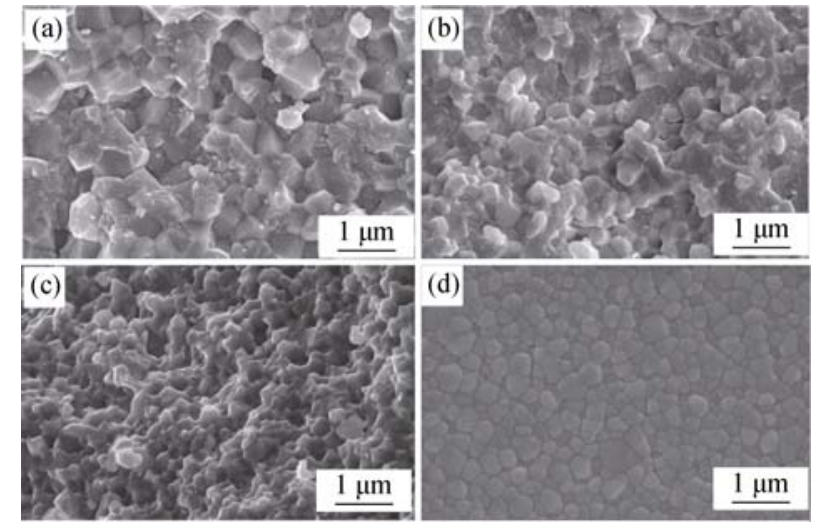

图 3 氧化锆的显微结构照片

Fig. 3 Morphologies of zirconia ceramics

(a) PS zirconia, fracture surface; (b) HP zirconia, fracture surface; (c) OPS zirconia, fracture surface; (d) OPS zirconia, polished surface

期晶粒生长的主要机制为晶界迁移, 控制晶粒生长 就需抑制晶界迁移机制, 强化晶界扩散、晶格扩散 等致密化机制。分析认为, 振荡压力强化了高温下 晶粒的塑性形变和晶界滑移等现象, 抑制了晶界迁 移, 从而抑制了氧化锆晶粒的过快生长。

\section{2 体积密度}

除了晶粒尺寸, 材料的致密度也是决定其力学 性能的重要参数。烧结过程中, 陶瓷材料晶界处的 残余闭气孔往往难以排出, 因此材料无法实现完全 致密化。图 4 对比了三种烧结方法所制备氧化锆的 相对密度, PS 氧化锆的相对密度仅为 $98.2 \%$; 施加 $30 \mathrm{MPa}$ 压力后, HP 氧化锆的致密度相比 PS 氧化锆 有较大的提高, 但 HP 氧化锆的孔隙率仍有 $0.8 \%$, 微观结构分析显示这些气孔主要以晶界处闭气孔的 形式存在, 而且难以通过常规参数优化手段加以消 除。当施加 $30 \mathrm{MPa}$ 振荡压力后, 氧化锆的体积密度 达到 99.7\%, 表明 OPS 烧结法能够有效促进晶界处 闭气孔的排出。

对于晶界处尤其是三角晶界处的闭气孔，常 规压力烧结方法往往难以排除, 因此采用 HP、 SPS 烧结材料的致密度一般小于 $99 \%$ 。而在 OPS 烧结过程中，振荡压力不仅诱发高频压力，而且 对粒子表面局部加热(临近、相互接触的粒子间的 摩擦作用)。由于纳米粉体尺寸较小，粉体表面热 量能够迅速传递到粒子中心, 在这样的高温下, 许多材料具有一定的塑性，所以粒子在振荡压力 下会发生明显的塑性形变。振荡压力下晶粒的塑 性形变及晶界滑移, 加速了晶界处气孔的运动及 合并, 从而使闭气孔加速排出, OPS 氧化锆具有较 高的致密度。

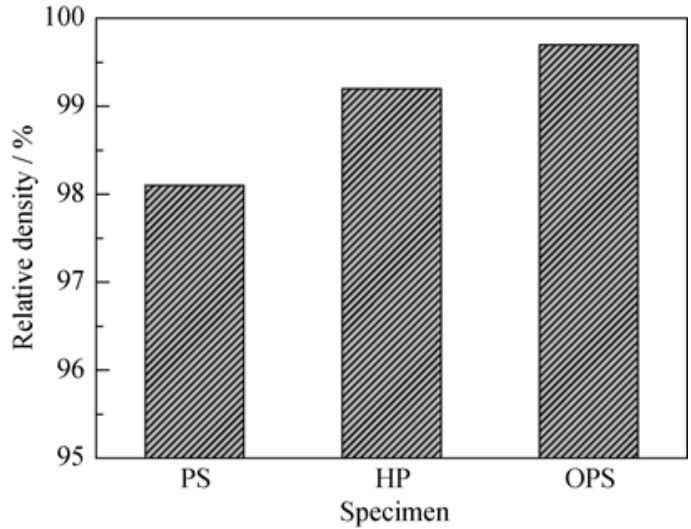

图 4 不同烧结方法所制备氧化锆陶瓷的相对密度变化

Fig. 4 Variation of relative density of zirconia sintered by different methods

\section{3 力学性能}

图 5 对比了三种烧结方式制备氧化锆陶瓷的 断裂强度, 三种材料的强度分别为 1003、1234 和 $1572 \mathrm{MPa}$ ，表明 OPS 法是制备高强度氧化锆陶瓷的 有效方法。结合前述分析, OPS 氧化锆的高强度源 于微观结构的变化: 一方面, OPS 氧化锆烧结过程 中有效排出了晶界处的闭气孔，材料的体积密度提 高到 99.7\%, 如图 4 所示; 另一方面, OPS 氧化锆烧 结过程中振荡压力抑制晶粒的过快生长, 同时将晶 粒尺寸抑制在较窄的尺寸区间内，如图 3 所示。根 据 Hall-Petch 关系式的描述，陶瓷中晶粒尺寸与强 度 $\sigma$ 间存在如下关系 ${ }^{[10]}$ :

$$
\sigma=\sigma_{0}+K d^{-0.5}
$$

其中 $\sigma_{0} 、 K$ 为常数, $d$ 为晶粒尺寸。由于振荡压 力产生的缺陷消除和晶粒细化效应, OPS 氧化锆 的断裂强度较相同压力的 HP 氧化锆提高了 $27.4 \%$, 可以满足诸多极端环境对高强度陶瓷材料 的需求。

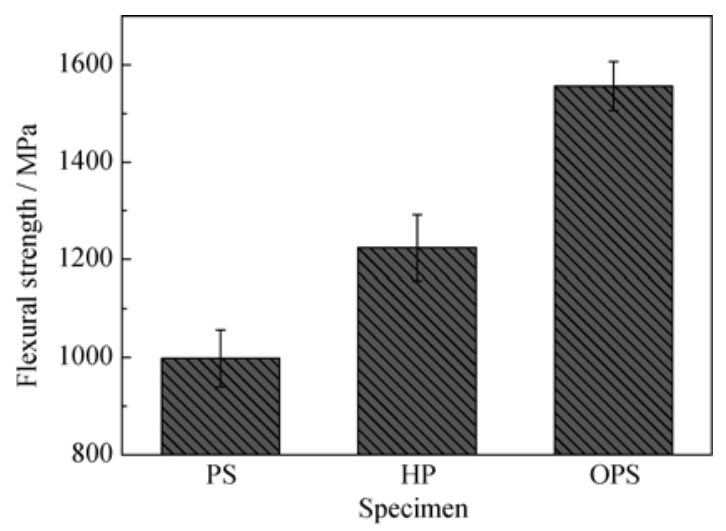

图 5 不同烧结方法制备氧化锆的抗弯强度变化

Fig. 5 Variation of flexural strength of zirconia by different sintering methods 
为了进一步研究烧结技术对氧化锆陶瓷断裂强 度可靠性的影响, 对三种氧化锆的断裂强度进行了 韦伯(Weibull)分布统计分析, 计算公式如下 ${ }^{[21,4]}$ :

$$
\ln \ln \left[\frac{1}{1-P_{n}}\right]=m \ln \sigma^{n}-m \ln \sigma^{0}
$$

$P_{n}$ 为断裂强度概率因子, 计算公式如下:

$$
P_{n}=(n-0.5) / N
$$

其中, $n$ 为排列序号, $N$ 为总测量样本数, $\sigma^{n}$ 为排 在第 $n$ 位的样品的断裂强度, $m$ 为韦伯模数。图 6 为三种氧化铅断裂强度的 Weibull 模数分布图, 研 究的样本数为 15 。PS 氧化锆的致密度较低, 材料中 闭气孔和微裂纹等缺陷浓度较高, 因此材料的可靠 性较低, Weibull 模数为 13.03。在烧结过程中施加外 力可以提高粉体扩散的烧结驱动力, 因此 HP 氧化 锆和 OPS 氧化锆断裂强度的 Weibull 模数分别达到 20.84 和 32.59, 表明其可靠性显著提高。

\section{4 低温老化性能}

所谓低温老化(Low Temperature Degradation, LTD) 是指 Y-TZP 陶瓷长时间处于 $100 \sim 400^{\circ} \mathrm{C}$ 的水热 环境下力学性能会发生严重下降的现象。低温老 化现象严重限制了 Y-TZP 材料在中低温的使用, 尤其是在潮湿和有水存在条件下的使用 ${ }^{[22]}$ 。按照 ISO13356-2008 标准, 实验开展了氧化锆在 $134^{\circ} \mathrm{C}$ 、 $0.2 \mathrm{MPa}$ 的水热环境中的老化行为研究, 老化时间 分别为 $6 、 12$ 和 $18 \mathrm{~h}$ 。图 7 为 HP、OPS 两种压力 烧结氧化锆断裂强度随老化时间的变化。老化前, HP 氧化锆和 OPS 氧化锆的断裂强度分别为 1234 和 $1572 \mathrm{MPa}$; 随着老化时间的延长, 两种氧化锆的强 度逐渐降低, 当老化时间为 $18 \mathrm{~h}$ 时, HP 氧化锆和



图 6 不同烧结方法制备氧化锆的 Weibull 模数变化

Fig. 6 Variation of Weibull modulus of zirconia by different sintering methods

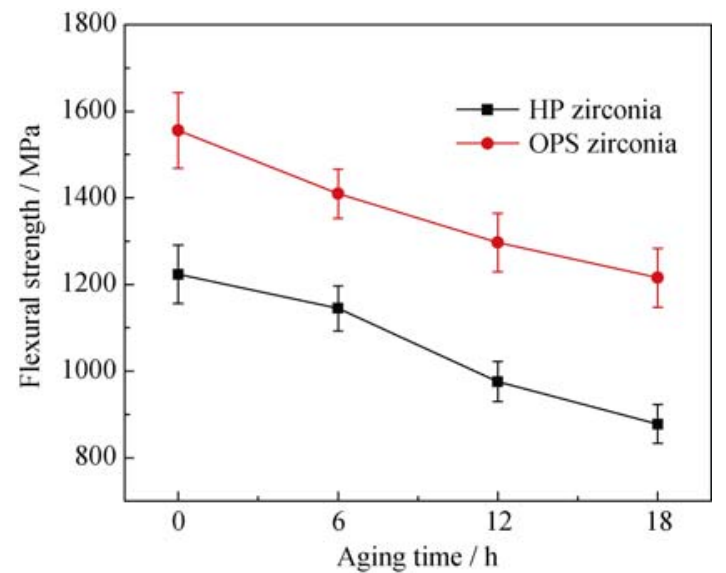

图 $7 \mathrm{HP}$ 氧化锆和 OPS 氧化锆的断裂强度随老化时间的变化 Fig. 7 Variation of flexural strength with increase of aging time of zirconia by different sintering methods

OPS 氧化铅的断裂强度分别为 878 和 $1106 \mathrm{MPa}$ 。在 老化过程中，尽管 OPS 氧化锆的强度出现了明显的 降低，但其强度始终高于 HP 氧化锆的强度。

\section{5 烧结机理}

综合上述研究, 无压烧结过程的驱动力是粉体 表面能的降低, 坏体致密化的烧结机理主要为蒸 发一凝聚、晶格扩散和晶界扩散等。热压烧结过程的 驱动力除了系统表面能外, 还有外加压力的作用, 其烧结机理包括粘性流动、塑性形变、晶界扩散 和颗粒重排等。与传统热压烧结法的恒定压力一 “死力” 不同，振荡压力烧结过程中施加的是振幅 与频率可控的 “活力”，赋予了材料多种全新的 烧结机制。

图 8 为热压烧结与振荡压力烧结条件下材料致 密化与晶粒生长过程对比图。振荡压力烧结过程中, 烧结开始前 (Stage I ), 振荡压力可以通过颗粒滑 移、旋转、破碎等机制促进颗粒重排和消除颗粒团 聚, 因此提高了素坏的堆积密度, 缩短了原子/离子 扩散路径; 烧结过程的前期和中期(Stage II ), 振荡 压力可以加速粘性流动和扩散蠕变, 激发晶界滑 移、塑性形变等机制，从而加速材料的致密化; 烧结 后期(StageIII), 振荡压力引起的塑性形变促进了晶 界处气孔的合并和排出, 特别是排出了三角和四角 晶界处闭气孔, 因此能够制备接近理论密度的陶 瓷材料。简而言之, 振荡压力烧结过程中材料的致 密化主要源于如下两方面的机制：一是表面能作 用下的晶界扩散、晶格扩散和蒸发一凝聚等传统机制; 二是振荡压力赋予的新机制，包括颗粒重排、晶界 滑移、塑性形变以及形变引起的晶粒移动、气孔排 出等。

随着纳米技术的发展，纳米粉体被认为是提高 


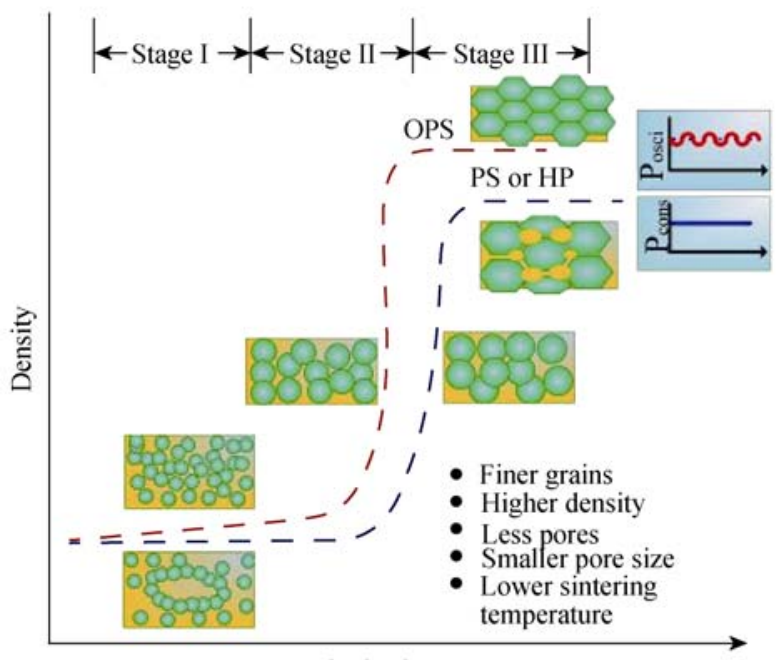

Grain size

图 8 恒定压力与振荡压力下陶瓷材料烧结路径示意图

Fig. 8 Schematic of sintering routes of ceramics under constant pressure and oscillatory pressure

结构陶瓷强度和可靠性的有效方法, 但是纳米粉体 粒径小、体系表面能较大, 颗粒极易发生团聚。采 用恒定压力烧结方法制备纳米材料时，高温下晶粒 迅速生长而粉体团聚无法及时消除，团聚体中的气 孔封闭后就无法排除。而采用振荡压力烧结技术, 动态载荷下团聚粉体极易打碎, 能够有效消除闭气 孔，有望制备性能优异的纳米陶瓷材料。

\section{3 结论}

采用振荡压力烧结法, 通过对待烧氧化锆粉料 施加 27.5 32.5 MPa 的振荡压力制备了高致密度、 细晶粒、高强度的氧化锆陶瓷。

1) 振荡压力加速了晶粒塑性形变和晶界滑移, 抑制了氧化锆晶粒的生长。统计表明 PS、HP 和 OPS 氧化锆的平均晶粒尺寸分别为 451、298 和 $251 \mathrm{~nm}$ 。

2) 振荡压力为粉体致密化提供较高的驱动力, 通过重排、扩散和迁移等机制加速了坏体的致密 化进程; 同时，振荡压力加速了烧结后期晶界处 闭气孔的排出。因此, OPS 氧化锆的相对密度达到 $99.7 \%$ 。

3) OPS 氧化锆致密度提高和晶粒细化提高了其 断裂强度和抗老化性能。与 PS 氧化锆相比, OPS 氧 化锆的断裂强度从 $1003 \mathrm{MPa}$ 提高到 $1572 \mathrm{MPa}$, Weibull 模数从 13.03 提高到 32.59 。

\section{参考文献:}

[1] KERN F. Ytterbia-neodymia-costabilized TZP-breaking the limits of strength toughness correlations for zirconia. J. Eur. Ceram. Soc., 2013, 33(5): 965-973.

[2] SHERMAN D, SCHLUMM D. Thickness effect in thermal shock of alumina ceramics. Scripta Materia., 2000, 42(8): 819-825.

[3] XUE W J, XIE Z P, LIU G W, et al. R-curve behavior of 3Y-TZP at cryogenic temperatures. J. Am. Ceram. Soc., 2011, 94 (9): 2775-2778.

[4] XUE W J, YI J, XIE Z P, et al. Enhanced fracture toughness of silicon nitride ceramics at cryogenic temperatures. Scripta Mater., 2012, 66(11): 891-894.

[5] RICHERSON D W. Modern Ceramic Engineering. New York: Marcel Dekker Inc, 1992: 130-150.

[6] 王否, 涂善东. 材料强韧学基础. 上海: 上海交通大学出版社, 2012: 105-156.

[7] BERNARD G, GUIZARD C. New relationships between relative density and grain size during solid-state sintering of ceramic powders. Acta Mater., 2008, 56(20): 6273-6282.

[8] OLEVSKY E A, TIKARA V, GRINO T. Multi-scale study of sintering: a review. J. Am. Ceram. Soc., 2006, 89(6): 1914-1922.

[9] LO WT, NAYAK P K, LU H H, et al. Evolution of binary phase $\mathrm{TiC} / \mathrm{Ti}_{3} \mathrm{SiC}_{2}$ composites from $\mathrm{TiC} / \mathrm{Ti} / \mathrm{Si}$ by hot-pressed reactive sintering. Mater. Sci. Eng: B-Adv., 2010, 172(1): 18-23.

[10] 谢志鹏. 结构陶瓷. 北京: 清华大学出版社, 2011: 213-223.

[11] SONG X C, LU J, ZHANG T S, et al. Two-stage master sintering curve approach to sintering kinetics of undoped and $\mathrm{Al}_{2} \mathrm{O}_{3}$-doped 8mol\% yttria-stabilized cubic zirconia. J. Am. Ceram. Soc., 2011, 94(4): 1053-1059.

[12] BERMARD G, ADDAD A, FANTOZZI G, et al. Spark plasma sintering of a commercially available granulated zirconia powder: comparison with hot-pressing. Acta Mater., 2010, 58(9): 3390-3399.

[13] EHRE D, GUTMANAS E Y, CHAIM R, et al. Densification of nanocrystalline MgO ceramics by hot-pressing. J. Eur. Ceram. Soc., 2005, 25(16): 3579-3585.

[14] XUE J X, LIU J X, XIE B H, et al. Pressure-induced preferential grain growth, texture development and anisotropic properties of hot pressed hexagonal boron nitride ceramics. Scripta Materia., 2011, 65(11): 966-969.

[15] JINKA A G K, LEWIS R W. Finite element simulation of hot isostatic pressing of metal powders. Comput. Method. Appl. M., 1994, 114(3/4): 249-272.

[16] WANG C, WANG X, ZHAO Z, et al. Microstructure homogeneity control in spark plasma sintering of $\mathrm{Al}_{2} \mathrm{O}_{3}$ ceramics. J. Eur. Ceram. Soc., 2011, 31(1/2): 231-235.

[17] XIE Z P, LI S, AN L N. A novel Ooscillatory pressure-assisted hot pressing for preparation of high performance ceramics. J. Am. Ceram. Soc., 2014, 97(4): 1012-1015.

[18] ACQUIER P, LEMONINIER S, ALLAIN B N, et al. Development of the dynamic compaction resistance sintering (DCRS): a new process for powder consolidation combining electric current and dynamic loading. J. Mater. Process Tech., 2015, 216: 447-454.

[19] CHEVALIER J, CALES B, DROUIN J M. Low-temperature aging of Y-TZP ceramics. J. Am. Ceram. Soc., 1999, 82(8): 2150-2154.

[20] DEVILLE S, GREMILLARD L, CHEVALIER J, et al. A critical comparison of methods for the determination of the aging sensitivity in biomedical grade yttria-stabilized zirconia. J. Biomed. Mater. Res. B, 2005, 72B(2): 239-245.

[21] YI J, XUE W J, XIE Z P, et al. Enhanced toughness and hardness at cryogenic temperature of silicon carbide sintered by SPS. Mat. Sci. Eng. A, 2013, 569: 13-17.

[22] ZINELIS S, THOMAS A, SYRES K, et al. Surface characterization of zirconia dental implants. Dent. Mater., 2010, 26(4): 295-305. 